\title{
PERFIL NUTRICIONAL DO CONSUMIDOR DE BARRAS DE CEREAIS
}

\section{NUTRITIONAL PROFILE OF THE CONSUMERS OF CEREAL BARS}

\author{
DEGÁSPARI, Cláudia Helena ${ }^{1^{*}}$; BLINDER, Elsa Wasserman ${ }^{2}$; MOTTIN, Fatima ${ }^{3}$ \\ ${ }^{1}$ Professora Adjunta da Universidade Tuiuti do Paraná. \\ Rua Com. Fontana, 405, ap. 803 - 80030-070 - Curitiba - PR \\ e-mail: sensus@cwb.matrix.com.br \\ ${ }^{2}$ Aluna de Graduação do Curso de Nutrição da Universidade Tuiuti do Paraná \\ e-mail: elsawb@ hotmail.com \\ ${ }^{3}$ Consultora Estatística da Empresa Painel Pesquisas. \\ e-mail: mottin_fatima@ @otmail.com
}

RECEBIDO: 05/02/08 ACEITO:11/03/08

\begin{abstract}
RESUMO:
O presente trabalho tem por finalidade descrever o perfil nutricional dos consumidores de barras de cereais em função do nível de atividade física praticada e as diversas intenções de consumo. Foram 232 entrevistados, sendo 148 consumidores de barras de cereais. Os resultados da análise indicam que a maioria dos consumidores demonstra interesse pelo sabor e textura do produto, sendo esses os principais motivos na escolha para o consumo. A maioria dos consumidores tem idade inferior a 44 anos e são do sexo feminino. Por ser um produto com preço relativamente alto, tem um consumo maior pela população com nível de renda alto. Ao analisar os indivíduos de acordo com o comportamento e hábitos alimentares, verifica-se que 0 grupo de pessoas eutróficas consome as barras como uma opção de lanche, o que demonstra ser um comportamento alimentar padrão. Já o grupo composto pelos indivíduos levemente desnutridos, observa-se um consumo para disfarçar a fome e por ser uma opção mais saudável, podendo ser um indício de transtorno alimentar. O grupo de pré-obesos apresenta um consumo parecido com o grupo citado anteriormente, isto é, consomem para disfarçar a fome, o que demonstra uma preocupação com a alimentação. Na análise realizada, percebe-se que o grupo de indivíduos com obesidade grau I, tem uma tendência de consumo diferente dos demais grupos analisados, onde o consumo é devido a regimes e dietas.
\end{abstract}

Palavras-chave: Perfil nutricional; barras de cereais; hábitos de consumo.

\section{ABSTRACT:}

The goal of this study is to describe the nutritional profile of the consumers of cereal bars with respect to their level of physical activity as well as their consumption intentions. Of the 232 subjects interviewed, 148 were consumers of cereal bars. The results show that most consumers are interested in the flavor and the texture of the bars. These are the main factors in the decisions to purchase cereal bars. Most consumers are women, younger than 44-years of age. Since it is a relatively expensive product, most consumers of cereal bars have a higher income level. When analyzing the data with respect to their behaviors and nutritional habits, the group characterized by normal body weight consumes cereal bars as a snack. The slightly mal-nourished group consumes cereal bars to hide their hunger or as a healthy option, which may point towards a nutritional disorder. The pre-obese group consumes cereal bars to, as mentioned in the previous group, hide their hunger, which demonstrates a degree of worry with what they eat. The obese I group is distinct from the other groups, as they consume cereal bars for dieting reasons.

Key-words: Nutritional profile, cereal bars, consumer behavior 


\section{INTRODUÇÃO}

As barrinhas definitivamente entraram para o cardápio brasileiro. Hoje se consomem quase seis vezes mais desses produtos do que há oito anos - são cerca de 500 milhões de unidades vendidas por ano. As preferidas são as de cereais com banana e as que têm chocolate. E é cada vez maior a variedade de marcas e tipos de "barrinhas de cereais", embora muitas nem tenham cereais na composição (APRILE, 2006).

Feitas a partir da compactação de frutas desidratadas e de cereais como a aveia, o trigo, a soja, o milho e o arroz, as barras de cereais ganharam grande popularidade no mercado; principalmente devido à sua praticidade, aos seus valores nutricionais e ao seu sabor. A barra é uma ótima opção para quem busca uma alimentação saudável nos lanches, ou também um complemento para refeições (UNIMED, 2007).

Por serem feitas de cereais, as barras possuem carboidratos, que são fontes energéticas para atividades físicas e mentais do dia-a-dia, vitaminas e minerais essenciais. E todas as marcas disponíveis no mercado fornecem um grupo variado de nutrientes, pois os ingredientes variam entre um produto e outro (UNIMED, 2007).

Para aqueles que desejam uma dieta de baixas calorias bem como saciar a necessidade de comer uma guloseima, esse alimento também é uma boa opção. Além de tudo, também ajuda a regular o intestino, contribuindo para o seu funcionamento (UNIMED, 2007).

Atualmente no mercado existem quatro tipos de barras: as fibrosas, as diet, as energéticas e as protéicas (APRILE, 2006; MACHADO, 2007; UNIMED, 2007).

As fibrosas possuem altos níveis de glicose e de fibras e, por fornecerem um nível considerável de energia, seu consumo é aconselhado após a prática de exercícios físicos. Também se recomenda a moderação no seu consumo devido ao excesso de fibras. Possuem em média $100 \mathrm{kcal}$ (APRILE, 2006; MACHADO, 2007; UNIMED, 2007).

Já as dietéticas ou diet têm menos calorias e gorduras e não contêm açúcar, portanto são adequadas para quem possui diabetes, ou deseja manter uma dieta com baixos níveis energéticos. Recomenda-se o consumo deste tipo de produto antes da prática de exercícios, desde que não contenham chocolate, devido ao teor lipídico. Essas barras não são recomendadas após a prática de exercícios, pois não são eficientes na reposição de grandes quantidades de energia. Possuem uma média de 65 kcal. (APRILE, 2006; MACHADO, 2007; UNIMED, 2007).

As energéticas são de fácil absorção, por serem menos fibrosas, mas possuem muitas calorias. Por isso devem ser consumidas durante ou após os exercícios e não são recomendadas para pessoas sedentárias. Muitas barras contêm chocolate e castanhas, granola e açaí. Em média possuem 280 kcal (APRILE, 2006; MACHADO, 2007; UNIMED, 2007).

As protéicas apresentam um menor teor lipídico e muita proteína, devendo ser consumidas após a atividade física com o objetivo do ganho de massa muscular. Não são aconselhadas para sedentários. Têm em torno de 200 kcal (APRILE, 2006; MACHADO, 2007; UNIMED, 2007).

Para permitir a agregação dos ingredientes, as barras costumam conter xarope de glucose, embora algumas usem mel para isso. Não é indicado nutricionalmente que a barra contenha mais xarope do que cereal, pois os dois são carboidratos, sendo que a diferença entre ambos é a velocidade de absorção pelo organismo. O xarope, um carboidrato simples, fornece uma energia rápida. Já o cereal, um carboidrato complexo, é absorvido mais lentamente e, portanto, a energia é contínua, de longo prazo. (APRILE, 2006).

O objetivo do trabalho é descrever o perfil nutricional dos consumidores de barras de cereais em função do nível de atividade física praticada e as diversas intenções de consumo. 
O trabalho se justifica à medida que irá diferenciar o comportamento alimentar dos consumidores de barras de cereais de acordo com o perfil nutricional de cada grupo analisado.

\section{MATERIAL E MÉTODOS}

Para o desenvolvimento deste trabalho, foi aplicado um estudo descritivo, utilizando um questionário com 13 perguntas fechadas encadeadas e apenas uma aberta (aprovado pelo Comitê de Ética em Pesquisa em Humanos e Animais da Universidade Tuiuti do Paraná), todas voltadas ao tema: consumo de barras de cereais, conforme apresentado no ANEXO 1. Neste questionário buscou-se identificar as razões que levam as pessoas a consumirem barras de cereais, embasado por uma análise estatística adequada.

A amostragem utilizada neste trabalho foi a de amostras não probabilísticas ou intencionais, que tentam reproduzir o mais fielmente possível a população, tendo sido utilizada por se apresentar mais prática, menos onerosa e mais rápida. Neste tipo de amostra o universo a ser estudado é dividido em subuniversos, e a amostra total é distribuída entre os subuniversos respeitando suas proporções. (TRIOLA, 1999).

Foram entrevistadas 232 pessoas ( $54 \%$ do sexo feminino e $46 \%$ do sexo masculino, conforme recomendado por DEGÁSPARI \& WASZCZYNSKYJ, 1998), entre consumidores e não consumidores de barras de cereais, sendo os locais de coleta dos dados na Universidade e empresas na cidade de Curitiba, tornando bem amplo o público alvo. Para o desenvolvimento do trabalho foram utilizados apenas os dados dos consumidores de barras de cereais, utilizando uma amostra de 148 pessoas. Os dados coletados foram analisados estatisticamente para que se tenha uma interpretação científica. (TRIOLA, 1999; AMERINE, PANGBORN \& ROESSLER, 1965).

Para análise da pesquisa foi utilizada estatística descritiva e teste qui quadrado $\left(\square^{2}\right)$. 0 teste $\square^{2}$ aplicável em dados representados em forma de freqüência, é utilizado para detectar significância estatística da diferença entre dois grupos independentes.

$$
\text { Estatística do teste teste } \Rightarrow \chi^{2}=\sum(\mathrm{O} \tilde{\mathrm{n}} \mathrm{E})^{2}
$$

$\mathrm{E}$

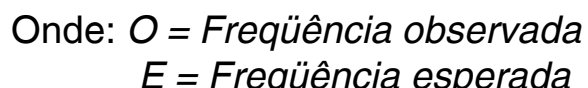

O teste c2 é uma estatística não paramétrica de fácil compreensão e muito usada para verificar se há diferença entre freqüências esperadas e freqüências observadas de duas ou mais categorias. No caso, a freqüência esperada é sempre aquela que determina a igualdade dos grupos. O teste conflita a freqüência esperada da observada, e se forem verificadas diferenças significativas entre as duas categorias, os grupos são considerados diferentes.

\section{DISCUSSÃO DOS RESULTADOS}

Antes de analisar apenas no grupo dos consumidores de barras de cereais, é importante verificar as variáveis sexo, faixa etária e salário de todos os entrevistados (consumidores e não consumidores de barras de cereais). De acordo com o QUADRO 01, observa-se que as mulheres têm uma tendência maior a consumir barras de cereais do que os homens. 
QUADRO 01 - CONSUMO DE BARRAS DE CEREAIS DOS ENTREVISTADOS DE ACORDO COM O SEXO.

\begin{tabular}{|c|c|c|c|c|c|c|}
\hline & \multicolumn{2}{|c|}{ Não consumidores } & \multicolumn{2}{c|}{ Consumidores } & \multicolumn{2}{c|}{ Total } \\
\hline Sexo & Citações & $\%$ & Citações & $\%$ & Citações & $\%$ \\
\hline Feminino & 33 & $26,2 \%$ & 93 & $\mathbf{7 3 , 8} \%$ & 126 & $100,0 \%$ \\
\hline Masculino & 51 & $48,1 \%$ & 55 & $51,9 \%$ & 106 & $100,0 \%$ \\
\hline Total & 84 & $36,2 \%$ & 148 & $63,8 \%$ & 232 & $100,0 \%$ \\
\hline
\end{tabular}

Em relação à faixa etária, pode-se observar através do QUADRO 02 que praticamente todas as faixas etárias consomem barras de cereais.

QUADRO 02 - CONSUMO DE BARRAS DE CEREAIS DOS ENTREVISTADOS DE ACORDO COM A FAIXA ETÁRIA.

\begin{tabular}{|c|c|c|c|c|c|c|}
\hline \multirow{2}{*}{ Faixa etária } & \multicolumn{2}{|c|}{ Não consumidor } & \multicolumn{2}{c|}{ Consumidor } & \multicolumn{2}{c|}{ Total } \\
\cline { 2 - 7 } & Citações & $\%$ & Citações & $\%$ & Citações & $\%$ \\
\hline 15 a 24 anos & 26 & $38,8 \%$ & 41 & $61,2 \%$ & 67 & $100,0 \%$ \\
\hline 25 a 34 anos & 26 & $34,2 \%$ & 50 & $65,8 \%$ & 76 & $100,0 \%$ \\
\hline 35 a 44 anos & 17 & $30,9 \%$ & 38 & $69,1 \%$ & 55 & $100,0 \%$ \\
\hline 45 a 55 anos & 11 & $47,8 \%$ & 12 & $52,2 \%$ & 23 & $100,0 \%$ \\
\hline Mais de 55 anos & 3 & $37,5 \%$ & 5 & $62,5 \%$ & 8 & $100,0 \%$ \\
\hline Total & 83 & $36,2 \%$ & 146 & $63,8 \%$ & 229 & $100,0 \%$ \\
\hline
\end{tabular}

De acordo com o QUADRO 03, nota-se que os entrevistados que apresentam maior poder aquisitivo consomem mais barras de cereais, podendo este produto ser considerado praticamente como de elite.

QUADRO 03 - CONSUMO DE BARRAS DE CEREAIS DOS ENTREVISTADOS DE ACORDO COM A RENDA MENSAL.

\begin{tabular}{|c|c|c|c|c|c|c|}
\hline \multirow{2}{*}{ Faixa de renda } & \multicolumn{2}{|c|}{ Não consumidores } & \multicolumn{2}{c|}{ Consumidores } & \multicolumn{2}{c|}{ Total } \\
\cline { 2 - 7 } & Citações & $\%$ & Citações & $\%$ & Citações & $\%$ \\
\hline Até 1 sm & 8 & $50,0 \%$ & 8 & $50,0 \%$ & 16 & $100,0 \%$ \\
\hline De 1 a 2 sm & 14 & $33,3 \%$ & 28 & $66,7 \%$ & 42 & $100,0 \%$ \\
\hline De 2 a 3 sm & 18 & $40,0 \%$ & 27 & $60,0 \%$ & 45 & $100,0 \%$ \\
\hline 3 a 5 sm & 15 & $29,4 \%$ & 36 & $70,6 \%$ & 51 & $100,0 \%$ \\
\hline De 5 a 10 sm & 17 & $48,6 \%$ & 18 & $51,4 \%$ & 35 & $100,0 \%$ \\
\hline De 10 a 20 sm & 8 & $25,8 \%$ & 23 & $\mathbf{7 4 , 2 \%}$ & 31 & $100,0 \%$ \\
\hline Mais de 20 sm & 1 & $16,7 \%$ & 5 & $\mathbf{8 3 , 3 \%}$ & 6 & $100,0 \%$ \\
\hline Total & 81 & $35,8 \%$ & 145 & $64,2 \%$ & 226 & $100,0 \%$ \\
\hline
\end{tabular}


Nas análises apresentadas a seguir, serão considerados apenas os consumidores de barras de cereais, com o intuito de descrever o perfil nutricional destes consumidores em função do nível de atividade física praticada e as diversas intenções de consumo.

Observando o QUADRO 04, onde os consumidores foram avaliados de acordo com o IMC ou Índice de Massa Corpórea, a maioria $(67,4 \%)$ encontra se eutrófica e um percentual de $25,2 \%$ encontra-se em estado de pré obesidade.

QUADRO 04 - CLASSIFICAÇÃO DO IMC DOS CONSUMIDORES DE BARRA DE CEREAIS.

\begin{tabular}{|c|c|c|}
\hline Classificação do IMC & Total & $\%$ \\
\hline Desnutrição Leve & 4 & $3,0 \%$ \\
\hline Desnutrição Grave & 1 & $0,7 \%$ \\
\hline Normal & 91 & $\mathbf{6 7 , 4 \%}$ \\
\hline Pré-obesidade & 34 & $\mathbf{2 5 , 2 \%}$ \\
\hline Obesidade 1 & 7 & $5,2 \%$ \\
\hline Obesidade 2 & 1 & $0,7 \%$ \\
\hline Obesidade 3 & 1 & $0,7 \%$ \\
\hline Total & 135 & $100,0 \%$ \\
\hline
\end{tabular}

Analisando o QUADRO 05, pode-se verificar que 70,9\% dos consumidores fazem com regularidade algum tipo de exercício físico.

\section{QUADRO 05 - PRÁTICA DE ATIVIDADE FÍSICA PELOS CONSUMIDORES DE BARRAS DE CEREAIS.}

\begin{tabular}{|c|c|c|}
\hline Grau de atividade física praticada & Total & $\%$ \\
\hline Sim, freqüento a academia & 28 & $\mathbf{1 8 , 9 \%}$ \\
\hline Sim, faço caminhadas regularmente & 23 & $\mathbf{1 5 , 5 \%}$ \\
\hline Às vezes faço exercício ou caminhada & 54 & $\mathbf{3 6 , 5 \%}$ \\
\hline Não faço exercício & 43 & $29,1 \%$ \\
\hline Total & 148 & $100,0 \%$ \\
\hline
\end{tabular}

Ao se analisar o motivo que leva as pessoas a consumirem as barras de cereais, verifica-se que $35,1 \%$ o fazem para disfarçar a fome, $27,0 \%$ por considerar o produto saudável, $26,4 \%$ utilizam-no na hora do lanche ou ainda $27,7 \%$ devido a sua praticidade, conforme observados no QUADRO 06. 
QUADRO 06 - MOTIVO QUE CONDUZ AO CONSUMO DE BARRAS DE CEREAIS.

\begin{tabular}{|c|c|c|}
\hline Motivo de consumo do produto & Total & $\%$ \\
\hline Para disfarçar a fome & 52 & $\mathbf{3 5 , 1 \%}$ \\
\hline Praticidade & 40 & $\mathbf{2 7 , 7 \%}$ \\
\hline Pode ser mais saudável & 40 & $\mathbf{2 7 , 0} \%$ \\
\hline Na hora do lanche & 39 & $\mathbf{2 6 , 4 \%}$ \\
\hline Por hábito & 23 & $15,5 \%$ \\
\hline Divido a regime e/ou dieta & 13 & $8,8 \%$ \\
\hline Para manter a forma & 7 & $4,7 \%$ \\
\hline Antes ou após exercício & 7 & $4,7 \%$ \\
\hline Por alguma doença & 1 & $0,7 \%$ \\
\hline Total & 148 & - \\
\hline
\end{tabular}

A escolha da marca ou tipo de barra de cereal é feita principalmente $(56,8 \%)$ devido ao sabor e textura do produto. O valor nutricional, que é o principal apelo promocional do produto, fica em segundo lugar com $23,6 \%$ dos consumidores, seguido pelo preço $(19,6 \%)$, conforme demonstrado no QUADRO 07.

QUADRO 07 - MOTIVO QUE INDUZ A ESCOLHA DA BARRA DE CEREAL.

\begin{tabular}{|c|c|c|}
\hline Motivos da escolha do produto & Total & $\%$ \\
\hline Pelo sabor e textura & 84 & $\mathbf{5 6 , 8 \%}$ \\
\hline Pelos valores nutricionais & 35 & $\mathbf{2 3 , 6 \%}$ \\
\hline Pelo preço & 29 & $\mathbf{1 9 , 6 \%}$ \\
\hline Pela marca & 12 & $8,1 \%$ \\
\hline Pela embalagem & 7 & $4,7 \%$ \\
\hline Pelo fabricante & 6 & $4,1 \%$ \\
\hline Total & 148 & - \\
\hline
\end{tabular}

Dividindo-se os consumidores de acordo com os seus perfis nutricionais, pode-se analisar cada grupo de acordo com o seu comportamento frente à prática de atividade física, ao motivo de consumo e ao motivo da escolha da marca/tipo de produto, conforme descrito nos itens a seguir.

\subsection{PERFIL NUTRICIONAL DE DESNUTRIÇÃO LEVE.}

Dentre os consumidores que apresentam um perfil de desnutrição leve (4 consumidores), verifica-se que todos são do sexo feminino e com idade inferior a 44 anos, sendo que 3 destas consumidoras disseram praticar algum tipo de atividade física e 2 utilizam o produto por o considerá-lo saudável ou disfarçar a fome, fazendo sua escolha pelo sabor e textura (QUADROS $08,09,10)$. 
QUADRO 08 - PRÁTICA DE ATIVIDADE FÍSICA PELO GRUPO COM DESNUTRIÇÃO LEVE.

\begin{tabular}{|c|c|c|}
\hline Grau de atividade física praticada & Total & $\%$ \\
\hline Sim, faço caminhadas regularmente & 1 & $25,0 \%$ \\
\hline Às vezes faço exercício ou caminhada & 2 & $50,0 \%$ \\
\hline Não faço exercício & 1 & $25,0 \%$ \\
\hline Total & 4 & - \\
\hline
\end{tabular}

QUADRO 09 - MOTIVO QUE CONDUZ O CONSUMO PELO GRUPO COM DESNUTRIÇÃO LEVE.

\begin{tabular}{|c|c|c|}
\hline Motivos de consumo do produto & Total & $\%$ \\
\hline Por ser mais saudável & 2 & $50,0 \%$ \\
\hline Para disfarçar a fome & 2 & $50,0 \%$ \\
\hline Total & 4 & - \\
\hline
\end{tabular}

QUADRO 10 - MOTIVO QUE INDUZ A ESCOLHA PELO GRUPO COM DESNUTRIÇÃO LEVE.

\begin{tabular}{|c|c|c|}
\hline Motivos da escolha do produto & Total & $\%$ \\
\hline Pelo sabor e textura & 3 & $75,0 \%$ \\
\hline Pelo preço & 1 & $25,0 \%$ \\
\hline Total & 4 & - \\
\hline
\end{tabular}

3.2 PERFIL NUTRICIONAL DE EUTROFIA.

No grupo dos consumidores eutróficos observa-se que $72,5 \%$ praticam algum tipo de atividade física, consomem preferencialmente o produto na hora do lanche $(28,6 \%)$ e o escolhem pelo sabor e textura (59,3\%), conforme observado nos QUADROS 11, 12 e 13.

\begin{tabular}{|c|c|c|}
\hline Grau de atividade física praticada & Total & $\%$ \\
\hline Sim, freqüento a academia & 19 & $20,9 \%$ \\
\hline Sim, faço caminhadas regularmente & 14 & $15,4 \%$ \\
\hline Às vezes faço exercício ou caminhada & 33 & $36,3 \%$ \\
\hline Não faço exercício & 25 & $27,5 \%$ \\
\hline Total & 91 & - \\
\hline
\end{tabular}


QUADRO 11 - PRÁTICA DE ATIVIDADE FÍSICA PELO GRUPO COM DOS EUTRÓFICOS. QUADRO 12 - MOTIVO QUE CONDUZ O CONSUMO PELO GRUPO DE EUTRÓFICOS.

\begin{tabular}{|c|c|c|}
\hline Motivos de consumo do produto & Total & $\%$ \\
\hline Na hora do lanche & 26 & $28,6 \%$ \\
\hline Pode ser mais saudável & 24 & $26,4 \%$ \\
\hline Para disfarçar a fome & 19 & $20,9 \%$ \\
\hline Por hábito & 14 & $15,4 \%$ \\
\hline Para manter a forma & 10 & $11,0 \%$ \\
\hline Divido a regime e/ou dieta & 7 & $7,7 \%$ \\
\hline Praticidade & 4 & $4,4 \%$ \\
\hline Por alguma doença & 1 & $1,1 \%$ \\
\hline Total & 91 & - \\
\hline
\end{tabular}

QUADRO 13 - MOTIVO QUE INDUZ A ESCOLHA PELO GRUPO DE EUTRÓFICOS.

\begin{tabular}{|c|c|c|}
\hline Motivos da escolha do produto & Total & $\%$ \\
\hline Pelo sabor e textura & 54 & $59,3 \%$ \\
\hline Pelos valores nutricionais & 24 & $26,4 \%$ \\
\hline Pelo preço & 17 & $18,7 \%$ \\
\hline Pela marca & 10 & $11,0 \%$ \\
\hline Pelo fabricante & 4 & $4,4 \%$ \\
\hline Pela embalagem & 1 & $1,1 \%$ \\
\hline Total & 91 & - \\
\hline
\end{tabular}

\subsection{PERFIL NUTRICIONAL DE PRÉ-OBESIDADE.}

No grupo com perfil nutricional de pré-obesidade, verifica-se que a maioria faz algum tipo de exercício $(70,6 \%)$, consomem o produto para disfarçar a fome $(32,4 \%)$ ou na hora do lanche $(20,6 \%)$, sendo que a escolha baseia-se nos atributos de sabor e textura $(52,9 \%)$, praticamente ignorando os fatores nutricionais do produto, conforme apresentado nos QUADROS 14, 15 e 16.

QUADRO 14 - PRÁTICA DE ATIVIDADE FÍSICA PELO GRUPO DE PRÉ OBESOS.

\begin{tabular}{|c|c|c|}
\hline Grau de atividade física praticada & Total & $\%$ \\
\hline Sim, freqüento a academia & 8 & $23,5 \%$ \\
\hline Sim, faço caminhadas regularmente & 4 & $11,8 \%$ \\
\hline Às vezes faço exercício ou caminhada & 12 & $35,3 \%$ \\
\hline Não faço exercício & 10 & $29,4 \%$ \\
\hline Total & 34 & - \\
\hline
\end{tabular}


QUADRO 15 - MOTIVO QUE CONDUZ O CONSUMO PELO GRUPO DE PRÉ-OBESOS.

\begin{tabular}{|c|c|c|}
\hline Motivos de consumo do produto & Total & $\%$ \\
\hline Para disfarçar a fome & 11 & $32,4 \%$ \\
\hline Na hora do lanche & 7 & $20,6 \%$ \\
\hline Pode ser mais saudável & 6 & $17,6 \%$ \\
\hline Divido a regime e/ou dieta & 5 & $14,7 \%$ \\
\hline Praticidade & 4 & $11,1 \%$ \\
\hline Por hábito & 3 & $8,8 \%$ \\
\hline Para manter a forma & 2 & $5,9 \%$ \\
\hline Antes ou após exercício & 1 & $2,9 \%$ \\
\hline Total & 34 & $100,0 \%$ \\
\hline
\end{tabular}

QUADRO 16 - MOTIVO QUE INDUZ A ESCOLHA PELO GRUPO DE PRÉ-OBESOS.

\begin{tabular}{|c|c|c|}
\hline Motivos da escolha do produto & Total & $\%$ \\
\hline Pelo sabor e textura & 18 & $52,9 \%$ \\
\hline Pelos valores nutricionais & 9 & $26,5 \%$ \\
\hline Pelo preço & 7 & $20,6 \%$ \\
\hline Pela marca & 2 & $5,9 \%$ \\
\hline Pelo fabricante & 1 & $2,9 \%$ \\
\hline Total & 34 & - \\
\hline
\end{tabular}

\subsection{PERFIL NUTRICIONAL DE OBESIDADE 1.}

No grupo de Obesidade 1, observa-se um comportamento diferente dos demais grupos supracitados. Neste grupo verifica-se que $42,9 \%$ não pratica nenhuma atividade física, porém $28,6 \%$ dos consumidores consomem este produto devido a necessidades de regime ou dietas. Mesmo assim, ainda o sabor e a textura é o principal motivo que induz a escolha do produto com $57,1 \%$.

QUADRO 17 - PRÁTICA DE ATIVIDADE FÍSICA PELO GRUPO DE OBESOS 1.

\begin{tabular}{|c|c|c|}
\hline Grau de atividade física praticada & Total & $\%$ \\
\hline Sim, freqüento a academia & 1 & $14,3 \%$ \\
\hline Sim, faço caminhadas regularmente & 2 & $28,6 \%$ \\
\hline Às vezes faço exercício ou caminhada & 1 & $14,3 \%$ \\
\hline Não faço exercício & 3 & $42,9 \%$ \\
\hline Total & 7 & - \\
\hline
\end{tabular}


QUADRO 18 - MOTIVO QUE CONDUZ O CONSUMO PELO GRUPO DE OBESOS 1.

\begin{tabular}{|c|c|c|}
\hline Motivos de consumo do produto & Total & $\%$ \\
\hline Divido a regime e/ou dieta & 2 & $28,6 \%$ \\
\hline Pode ser mais saudável & 2 & $28,6 \%$ \\
\hline Na hora do lanche & 1 & $14,3 \%$ \\
\hline Antes ou após exercício & 1 & $14,3 \%$ \\
\hline Praticidade & 1 & $14,3 \%$ \\
\hline Total & 7 & - \\
\hline
\end{tabular}

QUADRO 19 - MOTIVO QUE INDUZ A ESCOLHA PELO GRUPO DE OBESOS 1.

\begin{tabular}{|c|c|c|}
\hline Motivos da escolha do produto & Total & $\%$ \\
\hline Pelo sabor e textura & 4 & $57,1 \%$ \\
\hline Pelo preço & 1 & $14,3 \%$ \\
\hline Pelos valores nutricionais & 1 & $14,3 \%$ \\
\hline Pela embalagem & 1 & $14,3 \%$ \\
\hline Pela marca & 1 & $14,3 \%$ \\
\hline Total & 7 & - \\
\hline
\end{tabular}

\section{CONCLUSÃO}

Ao analisar os resultados da pesquisa, observa-se uma tendência maior de consumo de barras de cereais pelo grupo das mulheres e que a maioria dos consumidores de ambos os sexos têm idade inferior a 44 anos.

Por se tratar de um produto com um preço relativamente alto, a população com nível de renda mais baixo apresenta um consumo menor, podendo ser considerado como um produto de elite.

Quanto à análise dos grupos (levemente desnutridos, eutróficos, pré obesos e obesos I), observa-se que a maioria dos indivíduos dos grupos analisados, dá preferência a escolha do produto de acordo com as características sensoriais das mesmas (sabor e textura). Por ser um produto com preço relativamente alto, tem um consumo maior pela população com nível de renda alto. Ao analisar os indivíduos de acordo com o comportamento e hábitos alimentares, verifica-se que o grupo de pessoas eutróficas consome as barras como uma opção de lanche, o que demonstra ser um comportamento alimentar padrão. Já o grupo composto pelos indivíduos levemente desnutridos, observa-se um consumo para disfarçar a fome e por ser uma opção mais saudável, podendo ser um indício de transtorno alimentar. O grupo de pré-obesos apresenta um consumo parecido com o grupo citado anteriormente, isto é, consomem para disfarçar a fome, o que demonstra uma preocupação com a alimentação. Na análise realizada, percebe-se que o grupo de indivíduos com obesidade grau I, tem uma tendência de consumo diferente dos demais grupos analisados, onde o consumo é devido a regimes e dietas. 
Com isso, questiona se se esses indivíduos consomem as barras por opção, ou devido a uma indicação de um profissional ou outras informações obtidas em revistas e propagandas.

De acordo com o comportamento e hábitos alimentares pode-se verificar que os grupos são bem definidos ao serem analisados. Através de um estudo como este, pode-se chegar a dados importantes que muitas vezes são ocultados por indivíduos portadores de transtornos alimentares. Cabendo aos profissionais envolvidos no desenvolvimento e prescrição destes produtos um olhar mais atento a apelos comerciais que induzem o comsumodas barras de cereais.

\section{REFERÊNCIAS}

AMERINE, M. A. ; PANGBORN, R. M. ; ROESSLER, E. B. Principles of sensory evaluation of food. London: Academic Press, 1965.

APRILE, J. Barras que valem ouro. Viva Saúde on Line, ed. 35, nov., 2006. Disponível em: < http://revistavivasaude.uol.com.br/edicoes/35/artigo35776-1.asp>. Acesso em: 12 set. 2007.

DEGÁSPARI, C. H., WASZCZYNSKYJ, N. Avaliação das Diferenças de Acuidade Gustativa entre Indivíduos do Sexo Feminino e Masculino. Alimentação Humana. Lisboa, v.IV, p.19 27, 1998.

MACHADO, Roberto. Para que serve as barras de cereais? BemStar. Disponível em: <http://www.doceshop.com.br/blog/?p=157>. Acessado em 16 jan. 2007.

UNIMED. Barras de cereais: qual escolher? UNIMED. Disponível em: <http:// www.unimeds.com.br/preview/?cod=17216> . Acesso em 08 mai. 2007.

TRIOLA, M. Introdução à Estatística. 7a ed. São Paulo: L.T.C, 1999. 
ANEXO 1: QUESTIONÁRIO APLICADO NA PESQUISA (Aprovado pelo Comitê de Ética em Pesquisa em Humanos e Animais da Universidade Tuiuti do Paraná).

1) VOCÊ É CONSUMIDOR DE BARRAS DE CEREAIS?

( ) SIM ( ) NÃO POR QUE?

2) SEXO: ( ) FEMININO ( ) MASCULINO

3) ESTADO CIVIL ( ) SOLTEIRO ( ) CASADO ( ) DIVORCIADO
( ) VIVENDO JUNTOS
( ) OUTROS

4) FAIXA ETÁRIA: ( ) 15 A 24 ANOS ( ) 25 A 34 ANOS ( ) 35 A 44 ANOS

( ) 45 A 55 ANOS ( ) > 55 ANOS

5) PESO (Kg):

ALTURA (METROS):

$\mathrm{IMC}=$ $\mathrm{Kg} / \mathrm{m}^{2}$

6) RENDA: () ATÉ 1SM () DE 1 A 2 SM () DE 2 A 3 SM () DE 3 A 5 SM ( ) DE 5 A 10 SM ( ) DE 10 A 20 SM ( ) MAIS DE 20 SM

7) GRAU DE INSTRUÇÃO: ( ) 1ํ GRAU INCOMPLETO ( ) 1ํ GRAU COMPLETO ( ) 2 GRAU INCOMPLETO ( ) 2 GRAU COMPLETO ( ) 3 GRAU INCOMPLETO ( ) 3 GRAU COMPLETO ( ) NENHUMA FORMAÇÃO

8) VOCÊ FAZ ALGUM EXERCÍCIO FÍSICO?

( ) SIM, FREQÜENTO ACADEMIA ( ) SIM, FAÇO CAMINHADAS REGULARMENTE

( ) ÀS VEZES FAÇO EXERCÍCIO/CAMINHADA ( ) NÃO FAÇO EXERCICIO FÍSICO

9) VOCÊ COSTUMA CONSUMIR BARRA DE CEREAL (MÁXIMO 2 ALTERNATIVAS):
( ) DEVIDO A REGIME/DIETA
( ) PARA DISFARÇAR A FOME
( ) PARA MANTER A FORMA
( ) ANTES/APÓS EXERCÍCIO FÍSICO
( ) NA HORA DO LANCHE
( ) POR HÁBITO
( ) POR SER MAIS SAUDÁVEL
() POR ALGUMA DOENÇA
( ) PRATICIDADE
( ) OUTROS

10) ONDE VOCÊ OBTEM AS INFORMAÇÕES SOBRE ESSE PRODUTO?
( ) TV
( ) JORNAL
( ) REVISTA
( ) OUTROS
( ) INTERNET
( ) LIVROS
SUPERMERCADO/BAR

() OUTROS

11). COMO VOCÊ COSTUMA ESCOLHER SUA BARRA DE CEREAL?

( ) PELO PREÇO ( ) PELOS VALORES NUTRICIONAIS ( ) DESIGN DA EMBALAGEM ( ) PELA MARCA ( ) PELO SABOR/TEXTURA ( ) PELO FABRICANTE

12) QUAL É A BARRA QUE VOCÊ COSTUMA CONSUMIR COM FREQÜÊNCIA? 
13) ONDE COSTUMA COMPRAR SUA BARRA DE CEREAL?

( ) NO SUPERMERCADO ( ) EM BANCAS DE JORNAL ( ) FARMÁCIAS

( ) EM BARES E LANCHONETES ( ) MERCADINHO

14) O QUE FALTA NAS BARRAS DE CEREAIS QUE EXISTEM NO MERCADO? 